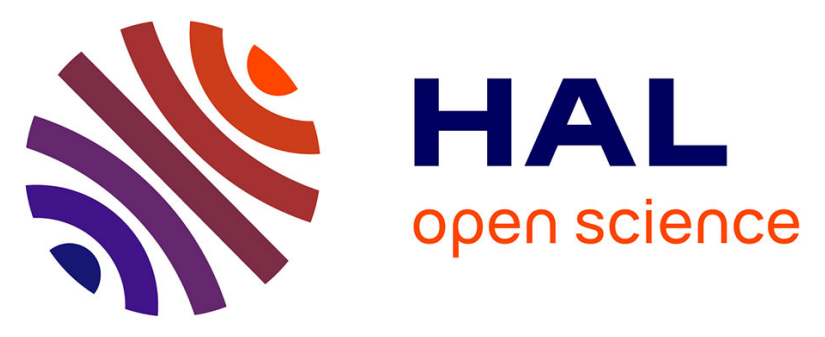

\title{
Determination of the Fracture Resistance of Ductile Polymers: The ESIS TC4 Recent Experience
}

Francesco Baldi, Silvia Agnelli, Luca Andena, Bamber Blackman, Leonardo

Castellani, Patricia Frontini, Jaroslav Kučera, Lucien Laiarinandrasana, Alessandro Pegoretti, Alicia Salazar, et al.

\section{To cite this version:}

Francesco Baldi, Silvia Agnelli, Luca Andena, Bamber Blackman, Leonardo Castellani, et al.. Determination of the Fracture Resistance of Ductile Polymers: The ESIS TC4 Recent Experience. Materials Performance and Characterization, 2020, 9 (5), pp.20190175. 10.1520/MPC20190175 . hal-03207111

\section{HAL Id: hal-03207111 \\ https://hal.science/hal-03207111}

Submitted on 30 Sep 2021

HAL is a multi-disciplinary open access archive for the deposit and dissemination of scientific research documents, whether they are published or not. The documents may come from teaching and research institutions in France or abroad, or from public or private research centers.
L'archive ouverte pluridisciplinaire HAL, est destinée au dépôt et à la diffusion de documents scientifiques de niveau recherche, publiés ou non, émanant des établissements d'enseignement et de recherche français ou étrangers, des laboratoires publics ou privés. 


\title{
Determination of the Fracture Resistance of Ductile Polymers: The ESIS TC4 Recent Experience
}

\author{
Francesco Baldi, ${ }^{1}$ Silvia Agnelli, ${ }^{2}$ Luca Andena, ${ }^{3}$ Bamber R. K. Blackman, ${ }^{4}$ \\ Leonardo Castellani, ${ }^{5}$ Patricia M. Frontini, ${ }^{6}$ Jaroslav Kučera, ${ }^{7}$ Lucien Laiarinandrasana, ${ }^{8}$ \\ Alessandro Pegoretti, ${ }^{9}$ Alicia Salazar, ${ }^{10}$ and Laurent Warnet ${ }^{11}$
}

1 Dipartimento di Ingegneria Meccanica e Industriale, Università degli Studi di Brescia, Via Branze 38, 25123 Brescia, Italy (Corresponding author), e-mail: francesco.baldi@unibs.it, (1) https://orcid.org/0000-00016174-4474

2 Dipartimento di Ingegneria Meccanica e Industriale, Università degli Studi di Brescia, Via Branze 38, 25123 Brescia, Italy

3 Dipartimento di Chimica, Materiali e Ingegneria Chimica "G. Natta," Politecnico di Milano, Piazza Leonardo da Vinci 32, 20133 Milano, Italy

\section{ABSTRACT}

Round-robin (RR) tests carried out under the direction of the Technical Committee 4, "Polymers, Polymer Composites and Adhesives," of the European Structural Integrity Society (ESIS TC4) showed that the multispecimen methodology employed for the construction of the crack growth resistance curve ( $J$ vs crack extension, $\Delta a$ ) of polymers often does not provide reliable data because of the uncertainties associated with the measurement of $\Delta a$. With this in mind, the ESIS TC4 attention has been more recently focused on the analysis of a testing scheme based on the load separation criterion, which does not require the measurement of $\Delta a$. With the aim to employ this new approach into a standardized procedure, the degree of reproducibility of the results obtainable with the application of this testing scheme to ductile polymers has been assessed by means of multilaboratory RR testing exercises that started in 2011. An ESIS TC4 reference draft protocol was prepared and 10 laboratories participated in the RR activities. The present work describes the load separation criterion-based testing procedure recently examined by ESIS TC4 and gives a summary of the results obtained in the RR activities, which appear encouraging.

\section{Keywords}

J-integral, load separation criterion, fracture resistance, ductile polymers 


\footnotetext{
${ }^{4}$ Department of Mechanical Engineering, Imperial College London, South Kensington Campus, London SW7 2AZ, UK

${ }^{5}$ Versalis S.p.A., Basic Chemicals and Plastics Research Center, Via Taliercio 14, 46100 Mantova, Italy

${ }^{6}$ Universidad Nacional de Mar del Plata, Instituto de Investigaciones en Ciencia y Tecnología de Materiales (INTEMA), Colón 10850 Mar del Plata, Argentina (B7606BVZ)

7 Unipetrol RPA, s.r.o. - Polymer Istitute Brno, odštěpný závod, Tkalcovská 36/2, 60200 Brno, Czech Republic

${ }^{8}$ MINES ParisTech, PSL Research University, MAT-Centre des Matériaux, CNRS UMR 7633, BP 87, Evry Cedex, France

${ }^{9}$ Dipartimento di Ingegneria Industriale, Polo Scientifico e Tecnologico "F. Ferrari," Università degli Studi di Trento, Via Sommarive 9, 38123 Trento, Italy

${ }^{10}$ Grupo de Durabilidad e Integridad Mecánica de Materiales Estructurales (DIMME), Escuela Superior de Ciencias Experimentales y Tecnología, Universidad Rey Juan Carlos, C/ Tulipán s/n, Móstoles, 28933 Madrid, Spain

${ }^{11}$ Faculty of Engineering Technology, University of Twente, PO Box 217, 7500 AE Enschede, the Netherlands
}

\section{Introduction}

To determine the low-rate fracture resistance of ductile polymers, for which the application of standard linear elastic fracture mechanics (LEFM) tests fails, the material crack growth resistance $\left(J_{\mathrm{R}}\right)$ curve ( $J$ vs crack extension, $\Delta a$ ), developed within the frame of elastic-plastic fracture mechanics (EPFM), is generally employed. This is usually constructed by means of a multispecimen approach (procedure developed by ESIS TC4, ${ }^{1}$ that is, the Technical Committee 4, "Polymers, Polymer Composites and Adhesives," of the European Structural Integrity Society, ${ }^{2}$ and ASTM D6068-10(2018), Standard Test Method for Determining J-R Curves of Plastic Materials ${ }^{3}$ ). Specific ESIS TC4 round-robin (RR) tests showed that the uncertainties associated with the measurement of $\Delta a$ often make this approach unreliable. Furthermore, in many cases, an initiation fracture resistance parameter, $J_{I C}$, cannot be obtained. With this in mind, the attention of ESIS TC4 has been recently paid to the analysis of a single-specimen testing scheme based on the load separation criterion (LSC), which does not require the measurement of $\Delta a$ (see Agnelli et al. ${ }^{4}$ ). This approach would (i) allow the determination of a material initiation fracture resistance parameter, $J_{I, \text {, im }}$, and (ii) provide a rough measure of $\Delta a$ produced during the fracture test in the plastic region. In order to assess the degree of reproducibility of the results obtainable with the application of this method, in view of its possible employment in a standardized procedure, a multilaboratory activity was begun in September 2011 under the direction of ESIS TC4, with Università degli Studi di Brescia (Italy) as the coordinating laboratory. Ten laboratories (indicated in the authors' affiliation list) participated in this activity, which was organized on three consecutive RR testing exercises (RR1 to RR3). RR1 consisted in a preliminary work aimed at setting the key points for the preparation of the reference draft testing protocol, ${ }^{5}$ which was prepared in 2013 and then further reviewed in 2015. In RR2 and RR3, this drafted protocol (called RR protocol hereafter) was applied to the fracture characterization of polymeric materials that exhibit a ductile behavior but different degrees of stiffness and strength (from standard tensile tests). The outcomes were used to enhance the robustness of the method and to improve the protocol itself. The examined materials were as follows: an acrylonitrile-butadiene-styrene (ABS) and a high-impact polystyrene (HIPS) in RR2 (the RR2 results are presented in Agnelli et al. ${ }^{4}$ ); and a rubber-toughened polybutylene terephthalate (RT-PBT) and a linear low-density polyethylene (LLDPE) in RR3. The present work describes the LSC-based testing procedure examined by ESIS TC4 and gives a summary of the results obtained during the RR activities.

\section{The LSC-based Testing Method}

The method described in the RR protocol, ${ }^{5}$ founded on the LSC proposed by Ernst, Paris, and Landes, ${ }^{6}$ was derived from Sharobeam and Landes' works published in the early $90 \mathrm{~s}$ on metals. ${ }^{7,8}$ The LSC assumes that, for a defined specimen geometry, material, and constraint, the load, $P$, recorded in a fracture test in the plastic region can be mathematically represented as the product of two independent functions, as equation (1) shows:

$$
P=G\left(\frac{b}{W}\right) \cdot H\left(\frac{u_{p l}}{W}\right)
$$

where $G(b / W)$ and $H\left(u_{p l} / W\right)$ are the geometry and the material deformation functions, respectively, $W$ is the specimen width, $b$ the specimen uncracked ligament length (that is 
$W-a$, with $a$ that indicates the crack length), and $u_{p l}$ is the plastic displacement (that is the plastic component of the total displacement, $u$ ). The applicability of the LSC to polymeric materials, during both blunting and crack propagation phase, has been demonstrated (see Bernal, Cassanelli, and Frontini, ${ }^{9,10}$ Bernal, Montemartini, and Frontini, ${ }^{11}$ Morhain and Velasco, ${ }^{12,13}$ Salazar and Rodríguez, ${ }^{14}$ and Baldi, Agnelli, and Riccó ${ }^{15}$ ).

The testing method described in the RR protocol" is based on the construction of the "load separation parameter curve," i.e., the $S_{s b}$ curve, from tests in single edge-notched bending (SE(B)) configuration (see fig. 1). It requires the execution of two tests: on a sharp-notched $(\mathrm{sN})$ specimen and on a blunt-notched $(\mathrm{bN})$ specimen. A schematic representation of a sharp and of a blunt notch is shown in figure 1. In the sN specimen, fracture propagation occurs, whereas in the bN specimen, crack growth is hindered. From the load, $P$, versus displacement, $u$, curves obtained from quasi-static tests on $\mathrm{sN}$ and $\mathrm{bN}$ specimens, the separation parameter, $S_{s b}$, is determined as follows:

$$
S_{s b}\left(u_{p l}\right)=\left.\frac{P_{s}}{P_{b}}\right|_{u_{p l}}
$$

where $P_{s}$ and $P_{b}$ are load values read on $P$ versus $u_{p l}$ curves of the $\mathrm{sN}$ and bN specimens, respectively, at a given value of plastic displacement, $u_{p l}$, which, in the protocol examined here, is determined for each specimen as:

$$
u_{p l}=u-P \cdot C_{0}
$$

where $C_{0}$ is the initial elastic compliance of the specimen. With reference to $u_{p l}$ determined according to equation (3), that is from $C_{0}$, it is worth pointing out that (i) for the $\mathrm{sN}$ specimen in the crack propagation phase, $u_{p l}$ data assume a nominal character in consideration of the fact that the true $u_{p l}$ data should be evaluated from the actual compliance, $C$, that increases during the crack growth phase but is not known (the determination of $C$ would require test interruption and the measurement of $\Delta a$ [see Appendix, where true $u_{p l}$ data determined from $C$ have been used for the verification of the LSC validity for RT-PBT]); (ii) in consideration of the viscoelastic nature of polymeric materials, $u_{p l}$ represents the "non-elastic" component of the total displacement, $u$, which for these materials is in general the sum of three contributions: elastic, viscoelastic, and plastic. Preliminary investigations specifically carried out by the laboratory of Brescia showed that, to the aims of the testing method examined here (RR protocol ${ }^{5}$ ), $u_{p l}$ data evaluated from $C_{0}$ can be successfully used.

It is expected that the $S_{s b}$ curve, that is, $S_{s b}$ plotted against $u_{p l}$, shows a "plateau" region, whereas $S_{s b}$ maintains an almost constant value $\left(S_{s b \text {,plateau }}\right)$, followed by a decreasing $S_{s b}$ region. The former region corresponds to the crack blunting phase, whereas the latter region corresponds to the crack propagation phase in the fracture process of the $\mathrm{s}$ specimen. The point between these two regions (called the limit point), at $u_{p l}=u_{p l \text { lim }}$, corresponds to fracture initiation (or pseudo-initiation, especially considering that for ductile polymers fracture initiation can be

FIG. 1

Schematic

representation of a SE

(B) specimen. $S$ is the

span. Sharp (of a sharp-

notched, sN, specimen)

and blunt (of a blunt-

notched, bN, specimen)

notches are also

represented; the blunt

notch is a keyhole type.
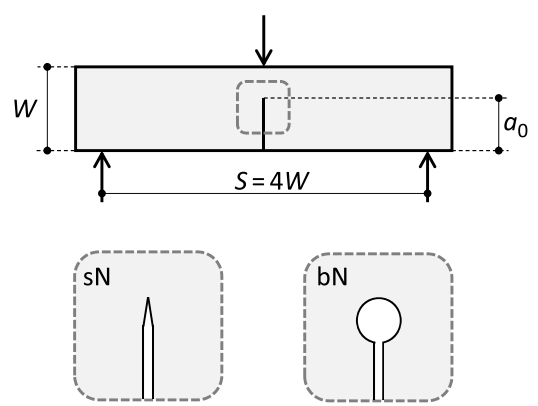
a complex progressive process ${ }^{16}$ ). J-integral value at $u_{p l, l i m}$, that is $J_{I, \text { lim }}$, which can be taken as a material pseudoinitiation fracture resistance parameter in place of the more conventional $J_{0.2}$ computed by the $J_{\mathrm{R}}$ curve (see the ESIS TC4 procedure $^{1}$ ), is evaluated as follows:

$$
J_{I, \lim }=\frac{2 \cdot U_{\text {lim,c }}}{B \cdot\left(W-a_{0}\right)}
$$

where $U_{\text {lim }, c}$ is the energy up to the limit point, corrected for indentation (see the ESIS TC4 procedure ${ }^{1}$ ), and $B, W$, and $a_{0}$ are the thickness, width, and initial crack length of the $\mathrm{sN}$ specimen, respectively.

Furthermore, the normalized separation parameter curve ( $R_{S}$ curve), i.e., $R_{S}$ versus $u_{p l}$, is traced $\left(R_{S}\right.$ is obtained as the ratio of $S_{s b}$ over $S_{s b \text {,plateau }}$ ), and the parameter $m_{S}$ is determined in the region of fracture propagation as follows:

$$
m_{S}=-\left.\frac{d R_{S}}{d u_{p l}}\right|_{u_{p l}>u_{p l \text { lim }}}
$$

It has been shown ${ }^{4}$ that parameter $m_{S}$, which is a specimen characteristic (i.e., dependent on both specimen geometry and material), provides an indication of the crack advancement produced per unit of $u_{p l}$ and could be used, as a "ductility index," to classify the fracture propagation processes by the amount of crack growth occurring within the plastic region. The higher the value of $m_{S}$, the higher the $\Delta a$ per unit of $u_{p}$ i if $m_{S} \rightarrow 0$, the process is governed by crack blunting.

Further details concerning this methodology can be found in Agnelli et al. ${ }^{4}$ and in the RR protocol. ${ }^{5}$

\section{Experimental}

Materials ABS, HIPS, and LLDPE were provided by Versalis SpA (Mantova, Italy), whereas RT-PBT was provided by Radici Novacips SpA (Villa d'Ogna, Bergamo, Italy). Table 1 reports the supply form of the materials, their basic mechanical properties and the nominal dimensions of the SE(B) specimens used in the RR fracture tests. For ABS, HIPS, and RT-PBT the fracture resistance, $J_{0.2}$ data are also indicated. They were obtained from the $J_{R}$ curve constructed by the application of the ESIS TC4 multispecimen approach ${ }^{1}$ on specimens having the same geometry and dimensions of the RR tests (for ABS and HIPS, $J_{0.2}$ data are from Agnelli et al. ${ }^{4}$; for RT-PBT, the $J_{R}$ curve is reported in the Appendix).

Each laboratory prepared and tested at least three $\mathrm{sN}$ and one $\mathrm{bN}$ specimens for each of the materials considered. The notching techniques were freely chosen by the laboratory, following the guidelines provided by the RR protocol. ${ }^{5}$ For LLDPE, two sets of specimens (\#a and \#b in Table 1) that differed in size were examined. This was done in order to explore, for this very ductile polymer (which also showed the lowest degrees of stiffness and

TABLE 1

Supply form of the materials examined and their basic mechanical properties (Young's modulus, E, and tensile yield stress,

\begin{tabular}{|c|c|c|c|c|c|c|c|}
\hline \multirow[b]{2}{*}{ Material (RR) } & \multirow[b]{2}{*}{ Supply Form } & \multicolumn{2}{|c|}{ Basic Mechanical Properties ${ }^{a}$} & \multicolumn{3}{|c|}{ SE(B) Specimen Dimensions ${ }^{\mathrm{b}}$} & \multirow[b]{2}{*}{$J_{0.2}\left(\mathrm{~kJ} / \mathrm{m}^{2}\right)$} \\
\hline & & $\mathrm{E}(\mathrm{MPa})$ & $\sigma_{y}(\mathrm{MPa})$ & B $(\mathrm{mm})$ & $\mathrm{W}(\mathrm{mm})$ & $a_{0}(\mathrm{~W})$ & \\
\hline HIPS (RR2) & Injection-molded dumbbells ${ }^{c}$ & 1,760 & 18 & 4 & 10 & 0.6 & 2.84 \\
\hline ABS (RR2) & Six-mm-thick compression molded plates & 2,500 & 44 & 6 & 12 & 0.6 & 5.71 \\
\hline RT-PBT (RR3) & Injection-molded dumbbells ${ }^{c}$ & 1,450 & 31 & 4 & 10 & 0.6 & 6.58 \\
\hline $\operatorname{LLDPE}^{\mathrm{d}}(\mathrm{RR} 3)$ & Ten-mm-thick injection-molded plates & 250 & 8 & 10 & $\begin{array}{l}10(\# \mathrm{a}) \\
20(\# \mathrm{~b})\end{array}$ & 0.6 & $-e^{e}$ \\
\hline
\end{tabular}
$\left.\sigma_{y}\right)$; nominal dimensions of $\mathrm{SE}(\mathrm{B})$ specimens used in the fracture tests; and $J_{0.2}$ data

Note: ${ }^{a}$ From quasi-static tests at room temperature. ${ }^{\mathrm{b}} \mathrm{B}$, thickness; $\mathrm{W}$, width; $a_{0}$, initial crack length (in bN specimen, notch tip radius of 1 mm); span used in fracture tests, $\mathrm{S}=4 \mathrm{~W}$ (ref. to fig. 1). ${ }^{\mathrm{c}}$ According to ISO 3167:2014, Plastics - Multipurpose Test Specimens; central narrow portion (with dimensions of $80 \times 10 \times 4 \mathrm{~mm}^{3}$ ) used for $\mathrm{SE}(\mathrm{B})$ specimen preparation. ${ }^{\mathrm{d}}$ Two sets of $\mathrm{SE}(\mathrm{B})$ specimens (\#a and \#b) used. ${ }^{\mathrm{e}}$ Not measured $\left(J_{R}\right.$ curve not constructed). 
strength among the materials examined), how the results obtainable by the application of the RR protocol could be affected by the specimen size (geometrical constraint). All the experiments were performed by means of universal testing machines at $\approx 23^{\circ} \mathrm{C}$ and with a crosshead rate of $1 \mathrm{~mm} / \mathrm{min}$. The data were processed according to the RR protocol, ${ }^{5}$ and the results (consisting of the $S_{s b}$ curve and data from $J_{I, l i m}$ and $m_{S}$, for each $s \mathrm{~N}$ specimen tested) were sent to the laboratory of Brescia for the comparative analysis.

The applicability of the LSC to styrenic polymers and polyolefins has been examined in several literature works; in contrast, to the best of the authors' knowledge, the applicability of the LSC to RT-PBT has never been checked. Therefore, before starting the RR activity on the RT-PBT, the validity of the LSC has been experimentally verified (see Appendix).

\section{Results and Discussion}

Figure $2 A$ and $2 B$ report $J_{I, \text { lim }}$ and $m_{S}$ results, respectively, for the various materials examined. Each datum reported is the mean value obtained by averaging all the data from the various laboratories. The datum considered for each laboratory is the average of the data obtained from the various $\mathrm{sN}$ specimens tested at the laboratory.

The degree of repeatability of the results within the same laboratory was generally higher than that of reproducibility (represented by the data of standard deviation reported in fig. 2). Results with a very high degree of repeatability could be obtained, and this emerges clearly from figure $3 A-E$, in which the $R_{S}$ curves of different nominally identical sN specimens of a given material, obtained at a given (selected) laboratory, are compared (the curves are vertically shifted for clarity). Figure $3 A$ refers to specimens of HIPS tested at Laboratory 10; figure $3 B$ refers to specimens of ABS tested at Laboratory 11; figure $3 C$ refers to RT-PBT tested at Laboratory 1 (the $R_{S}$ curves are reported both shifted and not); figure 3D and 3E refers to LLDPE specimens with size \#a and $\#$ b, respectively, tested at Laboratory 6 (laboratory numbers are in accordance with the authors' affiliation list). In figure $3 A$ and $3 B$, the $R_{S}$ curve indicated with an asterisk was built at Brescia based on the raw data provided by the laboratory that performed the tests. This was done, within the RR2 activities, just to check whether the data processing procedure described in the RR protocol ${ }^{5}$ had been properly applied by the laboratory. Figure $3 \mathrm{C}$

FIG. 2 Mean values ( \pm standard deviation) of $(A) J_{l, l i m}$ and $(B) m_{S}$ for the various materials examined, which were obtained by averaging all the (mean) data from the various laboratories (refer to Table 1 for the dimensions of the specimens). Values in parentheses indicate the standard deviation expressed as a percentage of the corresponding mean value. For LLDPE, valid $J_{l, \text { lim }}$ data could not be obtained (see text). Data with an asterisk are from Agnelli et al. ${ }^{4}$

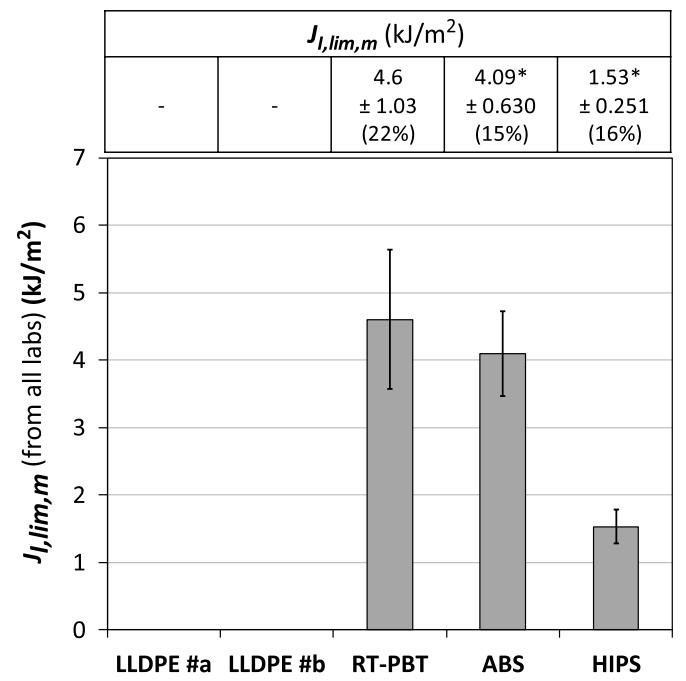

$(A)$

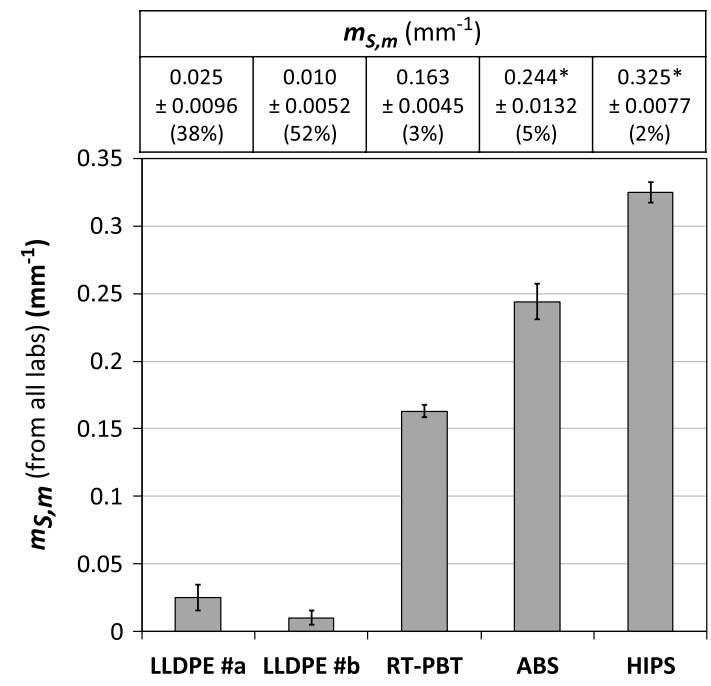

(B) 
FIG. 3 RS curves of different nominally identical sN specimens of (A) HIPS, from Laboratory 1O; (B) ABS, from Laboratory 11; (C) RT-PBT, from Laboratory 1; (D) and (E) LLDPE, \#a and \#b, respectively, from Laboratory 6 . The curves are vertically shifted by an $h$-factor. $\ln (A),(B)$, and $(C)$, the gray circles indicate the limit point. In $(C)$, the curves are also represented as not shifted (in gray); rhomb indicates the final point (that is, the point at which the test was interrupted). See text for explanation of the asterisk in $(A)$ and $(B)$.

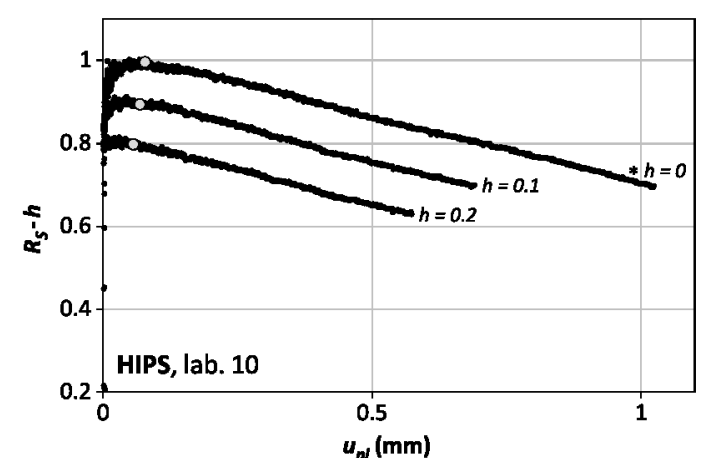

$(A)$

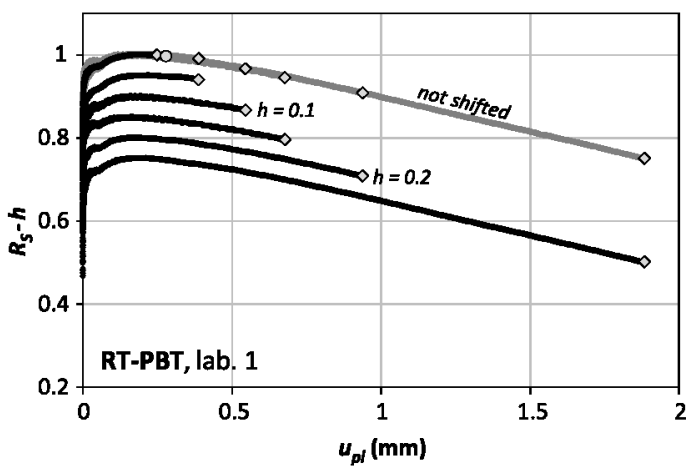

(C)

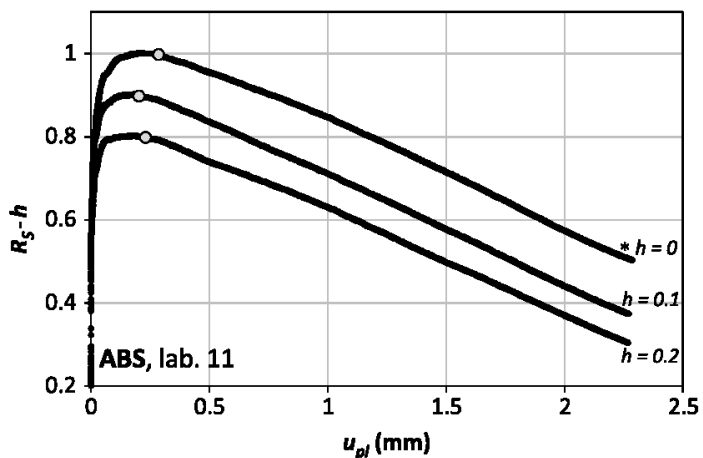

(B)

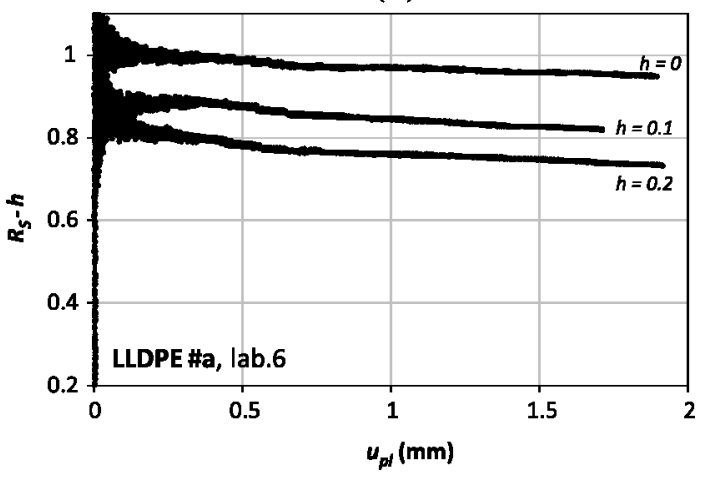

(D)

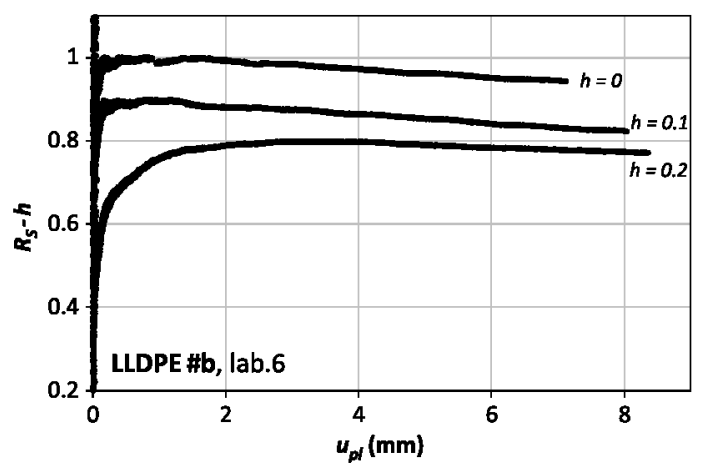

$(E)$

shows the $R_{S}$ curves obtained at Brescia (Laboratory 1) from six sN specimens of RT-PBT, tested up to different levels of displacement (the rhomb indicates, for each $\mathrm{sN}$ specimen test record, the point at which the fracture test was interrupted, i.e., the final point). If not shifted vertically, these curves overlap well, and they practically draw one single curve in which the plateau region that extends up to the limit point is clearly visible. For the LLDPE, the degree of repeatability of the results was generally lower with respect to the other materials, as can be observed in figure 3E. This is related to the fact that LLDPE was largely the most difficult-to-characterize material. Even if relatively high displacement values were reached (especially with size \#b; refer to fig. $3 E$ ), which is also necessary 
in consideration of the low Young's modulus of the material, reliable plateau regions could not be clearly identified in the $S_{s b}$ curves of the $\mathrm{sN}$ specimens tested. This indicates that, in the tests on the $\mathrm{SE}(\mathrm{B})$ specimens of the LLDPE examined herein, the crack blunting phase could not be distinguished from the crack propagation phase, and valid initiation fracture resistance data $\left(J_{I, \text { lim }}\right)$ could not be obtained.

Focusing attention on HIPS, ABS, and RT-PBT, for which data of both $J_{I, l i m}$ and $m_{S}$ have been obtained, it can be observed that the degree of reproducibility for the latter data is higher than that for the former data (see fig. $2 A$ and 2B). This suggests that, with respect to the fracture propagation phase, which is what the $m_{S}$ parameter refers to, fracture initiation/early stages of crack growth, which is what $J_{I, \text { lim }}$ refers to, are less reproducible (at a macroscopic scale). Examination of the results collected from the various laboratories seems to indicate that the scattering observed for the $J_{I, \text { lim }}$ data is related to a combination of both testing and data analysis aspects.

(i) In relation to testing, the results suggest that the quality of the notch of the $\mathrm{sN}$ specimens played an important role. As underlined by Salazar et al. ${ }^{17}$ and Martínez et al., ${ }^{18}$ the influence of the sharp notch quality on the determination of the initiation fracture resistance of polymeric materials, in plane strain conditions via both LEFM and EPFM methods, is still not fully understood. Within ESIS TC4, an RR exercise aimed at identifying the most suitable notching technique for the preparation of the notched specimens for the execution of plane strain LEFM tests is currently underway. ${ }^{2}$ The outputs of this RR activity will be used, at a later stage, as the basis for an investigation into the effects of the sharp notch quality in J-testing of ductile polymers.

(ii) With regard to data analysis, the results clearly point out that a crucial role is played by the determination of the initial specimen compliance, $C_{0}$. In addition, the procedure described in the RR protocol ${ }^{5}$ for the identification of the limit point on the $S_{s b}$ curve can be further improved. Within ESIS TC4, specific activities aimed at carefully examining these aspects are in progress. More specifically, an RR exercise aimed at evaluating the reproducibility degree of the data of initial elastic compliance, $C_{0}$, of $\mathrm{SE}(\mathrm{B})$ specimens of polymeric materials that exhibit a ductile behavior is currently underway.

Even if the degree of scattering observed for $J_{I, \text { lim }}$ data is higher than that of $m_{S}$ data, it can be acceptable within the field of fracture mechanics testing. Interestingly, not only for HIPS and ABS (as already pointed out by Agnelli et al. ${ }^{4}$ ) but also for RT-PBT, the $J_{I, \text { lim }}$ value is lower than the value of the technological $J_{0.2}$ parameter read on the $J_{R}$ curve (see Table 1 and Appendix). This suggests that the LSC-based approach examined here is able to provide data of fracture resistance that is more conservative with respect to $J_{0.2}$. It is worth noting, also, that for each material, the value of $J_{I, l i m}$ verified the size criteria proposed in the ESIS TC4 protocol ${ }^{1}$ for critical J-values, and this indicates that the $J_{I, \text { lim }}$ values refer to a plane strain state at the crack tip and that plasticity in the ligament is not excessive.

Figure 4 shows the $R_{S}$ curves constructed at the laboratory of Brescia in compliance with the RR protocol, for the SE(B) specimens of the different materials examined (one single curve for each material). For LLDPE, only the curve of size $\# \mathrm{~b}$, which refers to specimens with a width-to-thickness ratio closer to those of the specimens of the other materials, is reported. The differences among the fracture processes of the various specimens emerge clearly. Interestingly, by comparing the $R_{S}$ curves of RT-PBT and HIPS (that is, at fixed specimen dimensions, see Table 1), it can be observed that the extension of the plateau region of the curve is larger for the former than for the latter. This indicates that the higher fracture resistance obtained for RT-PBT with respect to HIPS ( $J_{\text {I,lim }}$ of RT$\mathrm{PBT}$ is $\approx 3$ times that of HIPS, see fig. $2 A$ ) can be related also to the fact that the blunting-to-fracture transition, in the fracture process of a sN specimen, occurs at a level of $u_{p l}$ that is higher for the former than for the latter.

The analysis of the fracture process based on the application of the RR protocol ${ }^{5}$ brings to light, for the LLDPE, a behavior remarkably different from that of the other ductile polymeric materials examined. For the construction of the $R_{S}$ curve of LLDPE, in absence of a reliable $S_{s b \text {,plateau }}$, following the protocol, ${ }^{5}$ the value

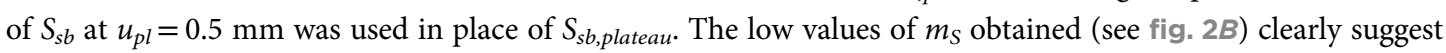
that the process is governed by blunting: irrespective of the specimen size considered, either \#a or \#b, crack growth produced per unit of $u_{p l}$ is very small. The high degree of scattering obtained for $m_{S}$ data suggests that its fracture process is not easily reproducible, contrary to what has been observed for the other materials for which standard deviations of $m_{S}$ are quite small. This is one of the reason why a reliable $J_{R}$ curve could not 
FIG. 4

$R_{S}$ curves obtained at Laboratory 1 from the

fracture tests on the

various materials

examined (one curve for

each material; refer to

Table 1 for the

dimensions of the

specimens). Gray circles

indicate the limit point on

the $R_{S}$ curves of HIPS,

$A B S$, and RT-PBT. For

each curve, $m_{S}$ is also

represented.

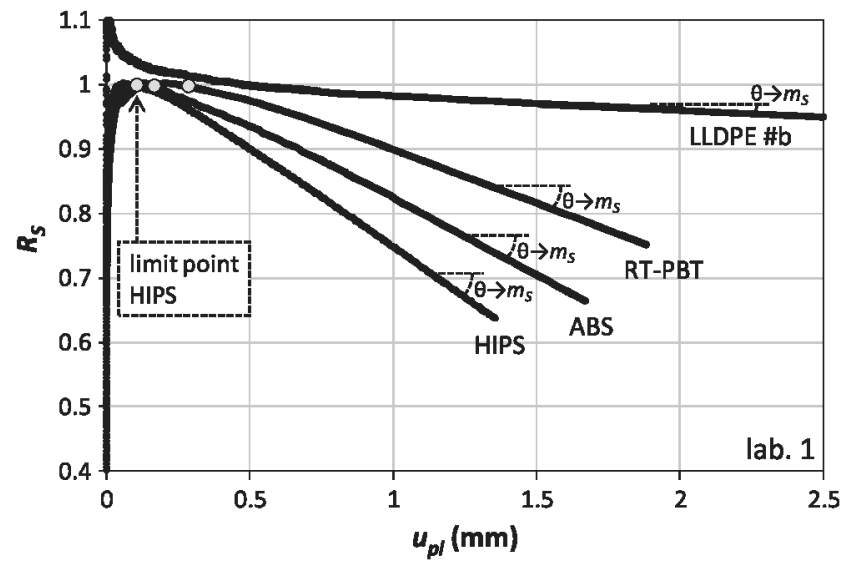

be constructed for this material (see Table 1), for which, furthermore, valid data of $\Delta a$ could not be obtained by the inspection of the fracture surface produced. Even if valid fracture resistance data $\left(J_{I, \text { lim }}\right)$ have not been determined for LLDPE, the testing procedure examined here was able to highlight, through $m_{S}$ determination, that a testing scheme based on the propagation of a crack cannot be successfully applied to this material, working on SE(B) specimens with dimensions as in Table 1. It would be necessary to use different specimen geometry/ dimensions or to resort to another testing approach that does not require the use of precracked specimens, such as cutting tests (see Patel, Blackman, and Williams ${ }^{19}$ ).

The results obtained for RT-PBT and LLDPE, in RR3, strengthen the idea that was outlined by Agnelli et al. $^{4}$ : to attribute a key role to the $m_{S}$ parameter in the fracture characterization of ductile polymers. The uncertainties associated with the measurement of $\Delta a$, which impair the construction of the $J_{R}$ curve, can have various causes: instrumental, methodological, or related to the intrinsic fracture behavior of the material. The measurement of $\Delta a$ is typically based on the optical analysis of the fracture surface generated during the test, and the greatest difficulty is associated with the correct evaluation of the crack front that defines the region on the fracture face characteristic of actual crack growth. More specifically, the obtainment of reliable $\Delta a$ data can be particularly arduous if, depending on the material, the crack growth produced in the fracture test is very limited or it is overshadowed by blunting (that was the case of the LLDPE examined here), or it is complicated by the occurrence of specific phenomena, such as with multiple cracking. Results obtained in the RR exercise reported here show that the application of the RR protocol, ${ }^{5}$ which in principle requires only two tests, is effective at distinguishing between, using the $m_{S}$ parameter, fracture processes governed by blunting $\left(m_{S} \rightarrow 0\right)$ and processes in which the crack growth actually occurs (with $m_{S}>0$ ). With this in mind, $m_{S}$ might be used as a key parameter in a criterion to check a priori if the application of the multispecimen approach for $J_{R}$ curve construction (founded on the measurement of $\Delta a$ ) to specimens with given geometry and dimensions is likely to fail. More specifically, with reference to $\mathrm{SE}(\mathrm{B})$ specimens (that is, the testing geometry considered in this work), a critical value of $m_{S}\left(m_{S, c}\right)$ might be fixed and used according to the following scheme:

- The LSC-based procedure is applied to a ductile polymer in the form of specimens with given dimensions, and the values of $m_{S}$ and $J_{I, l i m}$ are determined (the possibility that the $S_{s b}$ plateau region is not clearly identified and, therefore, that $J_{I, \text { lim }}$ is not evaluated cannot be ruled out);

- If $m_{S}>m_{S, c}$, then the value of $J_{I, \text { lim }}$ obtained is taken as the fracture resistance of the material (if the size criteria proposed for critical J-values in the ESIS TC4 protocol $^{1}$ are verified), and the application of the multispecimen approach for $J_{R}$ curve construction might be attempted; 
- If $m_{S} \leq m_{S, c}$, then $J_{I, l i m}$ (if determined) cannot be considered valid, and the multispecimen approach for $J_{R}$ curve construction cannot be applied; in this case, either the specimen dimensions are modified and new LSC-based tests performed or a different testing scheme that is not based on the use of precracked specimens (e.g., cutting tests ${ }^{19}$ ) is employed.

Of course, the analysis of the source of the errors in the measurement of $\Delta a$ deserves a special consideration. To this aim, specific ESIS TC4 activities are currently being developed. More specifically, interlaboratory RR exercises focused on the instrumental and methodological aspects related to the optical analysis of the fracture surface for $\Delta a$ evaluation will be organized. The possibility to resort to noncontact techniques (e.g., digital image correlation) for the indirect monitoring of crack advancement during the fracture test will also be considered.

\section{Conclusions}

The results obtained in the ESIS TC4 RR activity on the use of LSC in J-testing of ductile polymers are encouraging. The method under development has been successfully applied to ductile polymers that exhibit different degrees of stiffness and strength (ABS, HIPS, RT-PBT, and LLDPE). It allows determination of a material pseudoinitiation fracture resistance parameter $\left(J_{I, \text { lim }}\right)$ as well as a crack propagation parameter $\left(m_{S}\right)$. The results suggest that this latter parameter can play a key role in a criterion to check a priori if the multispecimen approach for J-testing (the ESIS TC4 procedure ${ }^{1}$ or the ASTM D6068-10(2018) ${ }^{3}$ ) applied to a ductile polymer (with given specimen geometry and dimensions) is likely to fail. The RR activity is still in progress, and special attention is being given to this latter outcome.

\section{ACKNOWLEDGMENTS}

The authors are grateful to Versalis SpA (Mantova, Italy) and Radici Novacips SpA (Villa d'Ogna, Bergamo, Italy) for kindly supplying the materials tested in this study.

\section{Appendix: Construction of $J_{R}$ Curve and Verification of the LSC Validity for RT-PBT}

The $J_{R}$ curve was constructed according to the ESIS TC4 procedure. ${ }^{1}$ The same specimen geometry and dimensions ( $\mathrm{sN}$ specimen, Table 1) and testing conditions (crosshead rate and temperature) of the RR tests were used. Figure A.1 shows the experimental $(\Delta a ; J)$ data points fitted by the power law best-fitting curve, the exclusion lines, and the blunting line, which was traced according to the following analytical expression:

$$
J=2 \cdot m \cdot \sigma_{y} \cdot \Delta a
$$

where a value of $m=1$ was used. The points on the $J_{R}$ fitting curve corresponding to $J_{0.2}$ (that is at $\Delta a=0.2 \mathrm{~mm}$ ) and to $J_{b l}$ (that is, at the intersection with the blunting line) are also indicated. The value of $J_{0.2}$ is reported in Table 1; $J_{b l}$ resulted in $1.39 \mathrm{~kJ} / \mathrm{m}^{2}$.

As underlined by Sharobeam and Landes, ${ }^{8}$ if the load is separable during the fracture propagation phase, $S_{s b}$ data evaluated at different levels of $u_{p l}$ (when the crack is growing) should lie on one single curve if plotted against the actual values of $b / W$. For such verification, the sN specimens that have the highest geometrical similarity among the specimens tested for the construction of the $J_{R}$ curve (which are those whose $R_{S}$ curves are reported in fig. $3 C$ ) were selected, and their $P$ versus $u$ curves and $\Delta a$ data at the final point were considered. In this analysis, differently from what was done for the application of the RR protocol, the actual values of $u_{p l}$ at the final points-where the crack length, $a$, is $a_{0}+\Delta a$-were used. These $u_{p l}$ data were evaluated from the actual specimen elastic compliance, $C(a / W)$, which was indirectly calculated by using the following $\operatorname{expression}^{20}$ : 
FIG. A.1 $J_{R}$ curve of RT-PBT, constructed according to the ESIS TC4 procedure. ${ }^{1}$ The experimental $(\Delta a ; J)$ data points (rhomb), the power law best-fitting curve (solid line), the exclusion lines (vertical dash-dot lines), the blunting line (dashed line), and the points on the fitting curve corresponding to $J_{b /}$ and $J_{0.2}$ (asterisk) —see text—are indicated. The equation of the power law best-fitting curve and the correlation coefficient of the fitting $\left(R^{2}\right)$ are also reported.

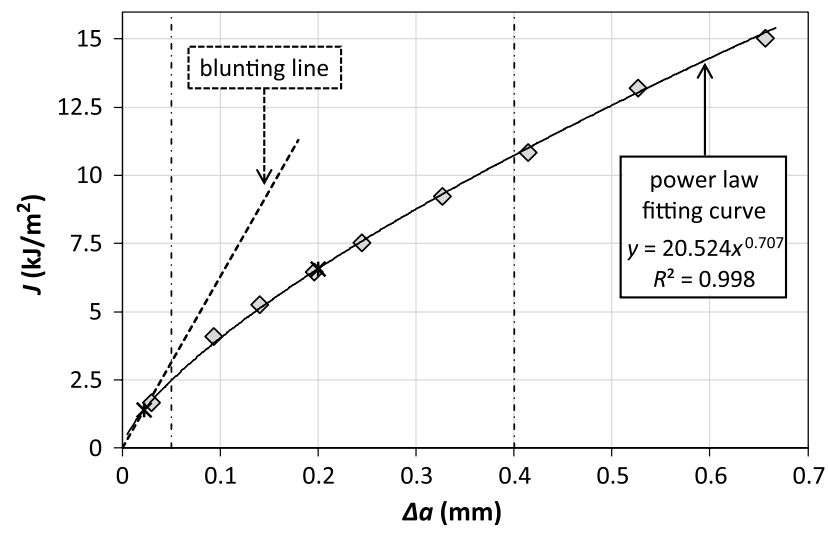

$$
C(a / W)=\frac{2 \cdot[f(a / W)]^{2} \cdot \Phi(a / W)}{E_{\text {fract }} \cdot B}
$$

where $f(a / W)$ and $\Phi(a / W)$ are tabulated functions. ${ }^{21}$ The value of $E_{\text {fract }}$, with a result of 1,400 $\pm 50 \mathrm{MPa}$, was previously determined by applying equation (A.2)—inverted—-to the data of $a_{0} / W$ and $C_{0}$ (corrected by taking into account the specimen indentation compliance; see the ESIS TC4 procedure ${ }^{1}$ ) of all the specimens used for the $J_{R}$ curve construction.

Figure A.2 shows the data points $\left(b / W ; S_{s b}\right)$ corresponding to the final points of the $\mathrm{sN}$ specimens test records considered. For $S_{s b}$ determination, the bN specimen test record of the RR experiments was used. These data points $\left(b / W ; S_{s b}\right)$, which are associated with different values of $u_{p l}$, draw a single trend, and this

FIG. A.2 Separation parameter, $S_{s b}$, evaluated at the final point of each $s N$ specimen test record of RT-PBT examined ( $R_{S}$ curves in fig. $3 C$ ), plotted against the corresponding value of the actual remaining ligament length divided by the specimen width, $b / W$ (rhomb). The dashed line indicates the power law best-fitting curve forced to the experimental $\left(b / W ; S_{s b}\right)$ data at the final points. The theoretical point with $S_{s b}=1$ (asterisk) - see text-is indicated. The equation of the fitting curve and the correlation coefficient of the fitting $\left(R^{2}\right)$ are also reported.

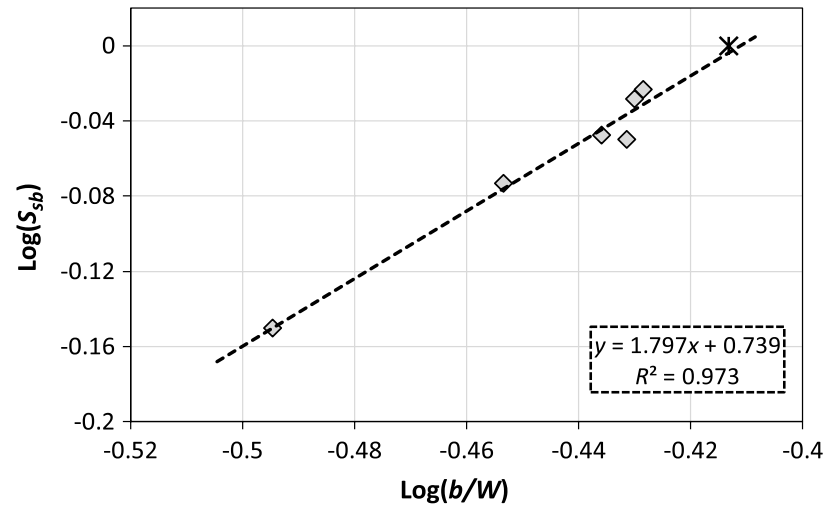


FIG. A.3 Normalized load, $P_{N}$, calculated at the final point of each sN specimen test record of RT-PBT examined ( $R_{S}$ curves in fig. 3C), plotted against the corresponding value of $u_{p} / W$ (rhomb). The dashed line indicates the power law best-fitting curve forced to the experimental data $\left(U_{p} / W_{;} P_{N}\right)$, whose equation and correlation coefficient $\left(R^{2}\right)$ are also reported. The solid line indicates the $P_{N}$ versus $u_{p} / W$ curve obtained from the bN specimen of RT-PBT.

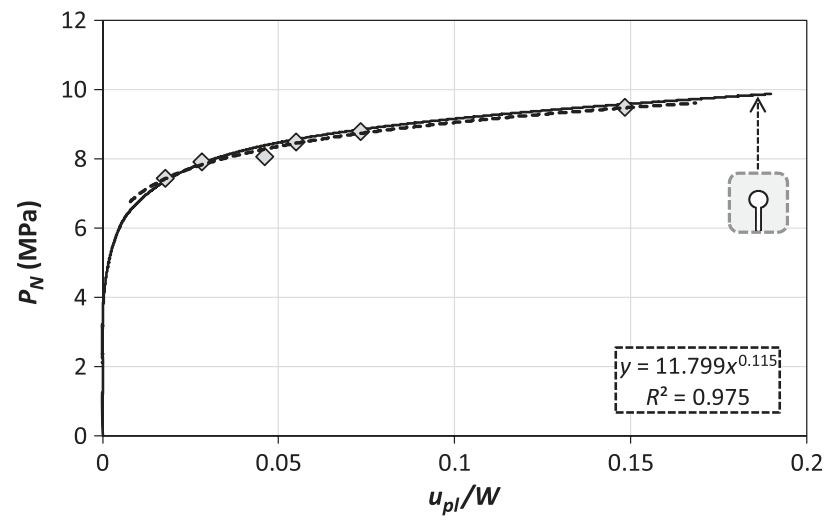

indicates that $u_{p l}$ has no contribution on the value of $S_{s b}$ and, therefore, that the LSC is valid during crack propagation. For $\mathrm{SE}(\mathrm{B})$ configuration, the geometry function, $G(b / W)$, can be expressed as (see Sharobeam and Landes $^{7}$ ) follows:

$$
G\left(\frac{b}{W}\right)=\left(\frac{b}{W}\right)^{\eta p l}
$$

Therefore, a power law best-fitting curve-linear in the bilogarithmic plot of figure A.2-was forced on the fracture propagation data points. According to equation (A.3), the slope of the fitting line is $\eta_{p}$, which results in 1.8. The difference between this value and the theoretical value of 2 derived by Rice, Paris, and Merkle ${ }^{22}$ for $\eta_{p l}$, and widely adopted in literature, is very similar to those observed in other literature works in which values of $\eta_{p l}$ were determined experimentally (see Sharobeam and Landes, ${ }^{8}$ Bernal, Montemartini, and Frontini, ${ }^{11}$ Baldi, Agnelli, and Riccò, ${ }^{15}$ and Baldi and Riccó ${ }^{23}$ ). Interestingly, the fitting curve passes very closely to the theoretical point at $S_{s b}=1$, which corresponds to a crack length in the $\mathrm{sN}$ specimen equal to the stationary crack length of the bN specimen.

The material deformation function, $H\left(u_{p l} / W\right)$, that characterizes the material during fracture propagation was then constructed-see equation (1). Following Sharobeam and Landes, ${ }^{8}$ it was built by referring to the normalized load, $P_{N}$, that was evaluated as follows:

$$
P_{N}\left(\frac{u_{p l}}{W}\right)=\frac{P\left(\frac{u_{p l}}{W}\right)}{B \cdot W \cdot G\left(\frac{b}{W}\right)}
$$

where $\eta_{p l}=1.8$ was used in the expression of $G(b / W)$. Figure A.3 shows the values of $P_{N}$ calculated at the final point of each $\mathrm{sN}$ specimen test record, plotted against the corresponding values of $u_{p} / W$. A power law best-fitting curve was forced to the experimental data points (this curve is known as "material key curve"; see Agnelli et al. ${ }^{24}$ ). In figure A.3, the $P_{N}$ versus $u_{p l} / W$ curve obtained from the $P$ versus $u$ curve of the bN specimen, which exhibits only blunting, is also reported. Interestingly, the material deformation function during the fracture propagation phase has the same form as during the blunting phase, and this is the experimental verification of the assumption on which the LSC is founded. 


\section{References}

1. G. E. Hale and F. Ramsteiner, "J-Fracture Toughness of Polymers at Slow Speed," in Fracture Mechanics Testing Methods for Polymers, Adhesives and Composites, European Structural Integrity Society Publication 28, ed. D. R. Moore, A. Pavan, and J. G. Williams (Oxford, UK: Elsevier, 2001), 123-157.

2. European Structural Integrity Society, "ESIS Home Page," European Structural Integrity Society, http://web.archive.org/ web/20200118124803/https:/www.structuralintegrity.eu/

3. Standard Test Method for Determining J-R Curves of Plastic Materials, ASTM D6068-10 (2018) (West Conshohocken, PA: ASTM International, approved October 1, 2018), https://doi.org/10.1520/D6068-10R18

4. S. Agnelli, F. Baldi, B. R. K. Blackman, L. Castellani, P. M. Frontini, L. Laiarinandrasana, A. Pegoretti, M. Rink, A. Salazar, and H. A. Visser, "Application of the Load Separation Criterion in J-Testing of Ductile Polymers: A Round-Robin Testing Exercise," Polymer Testing 44 (July 2015): 72-81, https://doi.org/10.1016/j.polymertesting.2015.03.019

5. S. Agnelli and F. Baldi, A Testing Protocol for the Construction of the Load Separation Parameter Curve for Plastics, ESIS TC4 Communication (European Structural Integrity Society, 2015).

6. H. A. Ernst, P. C. Paris, and J. D. Landes, "Estimations on J-Integral and Tearing Modulus T from a Single Specimen Test Record," in Fracture Mechanics, ed. R. Roberts (West Conshohocken, PA: ASTM International, 1981), 476-502, https:// doi.org/10.1520/STP28814S

7. M. H. Sharobeam and J. D. Landes, "The Load Separation Criterion and Methodology in Ductile Fracture Mechanics," International Journal of Fracture 47, no. 2 (January 1991): 81-104, https://doi.org/10.1007/BF00032571

8. M. H. Sharobeam and J. D. Landes, "The Load Separation and $\eta_{\mathrm{pl}}$ Development in Precracked Specimen Test Records," International Journal of Fracture 59, no. 3 (February 1993): 213-226, https://doi.org/10.1007/BF02555184

9. C. R. Bernal, A. N. Cassanelli, and P. M. Frontini, "A Simple Method for J-R Curve Determination in ABS Polymers," Polymer Testing 14, no. 1 (1995): 85-96, https://doi.org/10.1016/0142-9418(95)90616-O

10. C. R. Bernal, A. N. Cassanelli, and P. M. Frontini, "On the Applicability of the Load Separation Criterion to Acrylonitrile/ Butadiene/Styrene Terpolymer Resins,” Polymer 37, no. 18 (September 1996): 4033-4039, https://doi.org/10.1016/00323861(96)00232-7

11. C. R. Bernal, P. E. Montemartini, and P. M. Frontini, "The Use of Load Separation Criterion and Normalization Method in Ductile Fracture Characterization of Thermoplastic Polymers," Journal of Polymer Science Part B: Polymer Physics 34, no. 11 (August 1996): 1869-1880, https://doi.org/10.1002/(SICI)1099-0488(199608)34:11<1869::AID-POLB4>3.0.CO; $2-\mathrm{N}$

12. C. Morhain and J. I. Velasco, "Determination of J-R Curve of Polypropylene Copolymers Using the Normalization Method," Journal of Materials Science 36, no. 6 (March 2001): 1487-1499, https://doi.org/10.1023/A:1017500930544

13. C. Morhain and J. I. Velasco, "J-R Curve Determination of Magnesium Hydroxide Filled Polypropylene Using the Normalization Method," Journal of Materials Science 37, no. 8 (April 2002): 1635-1644, https://doi.org/10.1023/A: 1014944729912

14. A. Salazar and J. Rodríguez, "The Use of the Load Separation Parameter $\mathrm{S}_{\mathrm{pb}}$ Method to Determine the J-R Curves of Polypropylenes," Polymer Testing 27, no. 8 (December 2008): 977-984, https://doi.org/10.1016/j.polymertesting.2008. 08.013

15. F. Baldi, S. Agnelli, and T. Riccò, "On the Applicability of the Load Separation Criterion in Determining the Fracture Resistance (J Ic) of Ductile Polymers at Low and High Loading Rates," International Journal of Fracture 165, no. 1 (September 2010): 105-119, https://doi.org/10.1007/s10704-010-9510-9

16. F. Baldi, S. Agnelli, and T. Riccò, "On the Determination of the Point of Fracture Initiation by the Load Separation Criterion in J-Testing of Ductile Polymers," Polymer Testing 32, no. 8 (December 2013): 1326-1333, https://doi.org/ 10.1016/j.polymertesting.2013.08.007

17. A. Salazar, J. Rodríguez, F. Arbeiter, G. Pinter, and A. B. Martínez, "Fracture Toughness of High Density Polyethylene: Fatigue Pre-cracking versus Femtolaser, Razor Sharpening and Broaching," Engineering Fracture Mechanics 149 (November 2015): 199-213, https://doi.org/10.1016/j.engfracmech.2015.07.016

18. A. B. Martínez, A. Salazar, N. León, S. Illescas, and J. Rodríguez, "Influence of the Notch-Sharpening Technique on Styrene-Acrylonitrile Fracture Behavior,” Journal of Applied Polymer Science 133, no. 32 (August 2016): 43775, https://doi.org/10.1002/app.43775

19. Y. Patel, B. R. K. Blackman, and J. G. Williams, "Determining Fracture Toughness from Cutting Tests on Polymers," Engineering Fracture Mechanics 76, no. 18 (December 2009): 2711-2730, https://doi.org/10.1016/j.engfracmech.2009.07. 019

20. J. G. Williams, "Introduction to Linear Elastic Fracture Mechanics," in Fracture Mechanics Testing Methods for Polymers Adhesives and Composites, European Structural Integrity Society Publication 28, ed. D. R. Moore, A. Pavan, and J. G. Williams (Oxford, UK: Elsevier, 2001), 3-10.

21. J. G. Williams, Fracture Mechanics of Polymers (Chichester, UK: Ellis Horwood Limited, 1987).

22. J. R. Rice, P. C. Paris, and J. G. Merkle, "Some Further Results of J-Integral Analysis and Estimates," in Progress in Flaw Growth and Fracture Toughness Testing, ed. J. G. Kaufman, J. Swedlow, H. Corten, J. Srawley, R. Heyer, E. Wessel, and G. Irwin (West Conshohocken, PA: ASTM International, 1973), 231-245, https://doi.org/10.1520/ STP49643S 
23. F. Baldi and T. Riccò, "High-Rate J-Testing of Toughened Polyamide 6/6: Applicability of the Load Separation Criterion and the Normalization Method," Engineering Fracture Mechanics 72, no. 14 (September 2005): 2218-2231, https://doi. org/10.1016/j.engfracmech.2005.02.002

24. S. Agnelli, F. Baldi, L. Castellani, K. Pisoni, M. Vighi, and L. Laiarinandrasana, "Study of the Plastic Deformation Behaviour of Ductile Polymers: Use of the Material Key Curves," Mechanics of Materials 117 (February 2018): 105-115, https://doi.org/10.1016/j.mechmat.2017.11.002 